\title{
Erratum to: Feelings and Emotion-Based Learning
}

\author{
Jennifer A. Hawkins
}

\section{Erratum to:}

J.A. Hawkins, Feelings and Emotion-Based Learning, DOI 10.1007/ 978-3-319-66056-1

The initially published affiliation for the book author (City name) was incorrect. The correct Affiliation is as follows:

Jennifer A. Hawkins

Manchester Metropolitan University

Manchester, United Kingdom

Also, location has been removed from the end of the Preface.

The updated original online version for this book can be found at DOI 10.1007/978-3-319-66056-1 